\title{
Virtual Environments for Industrial Applications
}

\author{
Duncan Stevenson, Kevin Smith, Paul Veldkamp, John McLaughlin, Rochelle \\ O'Hagan, Dione Smith, Chris Gunn
}

\author{
CSIRO Mathematical and Information Sciences \\ GPO Box 664 Canberra ACT 2601 \\ Australia \\ duncan.stevenson@cmis.csiro.au
}

\begin{abstract}
A small-scale immersive virtual environment system (Virtual Workbench) and a haptic forcefeedback device (PHANToM) are used to show direct 3-dimensional interaction with fully 3-dimensional data. Real scenarios are used to show the value of the fully 3-dimensional interaction using both haptic and non-haptic devices.
\end{abstract}

KEYWORDS virtual environment, haptic

\section{INTRODUCTION}

The use of Virtual Environment systems for the display of and interaction with industrial and scientific data is about to become widely accepted in many industries which previously relied on older technologies such as printed plans and sections or 2-dimensional display and interaction techniques on a computer screen. The systems demonstrated here are commercially available products, at prices that are becoming affordable, and the time is ripe for serious consideration of the $\mathrm{HCI}$ research and advanced development that will lead to marketable products.

These systems focus on enhancing direct human sensory perception in the data interaction process. The Virtual Workbench (Poston, 1994) allows the user to put his or her hands directly into the data. The user perceives that the data and the hand-held location tools are in the same place, and can therefore make use of the hand and arm muscle receptors to locate and operate on the data. application situation. For example, in a medical situation they might be a scalpel or tweezers; in a mine modelling situation they might be a selector or a drawing tool.

The PHANToM (Massie, 1994) haptic device gives the user both a $3 \mathrm{D}$ locating device and also feedback on the "feel" of the data. This sensory feedback will apply to quite different metaphors. For instance, in simulating a medical application - say inserting a needle into human tissue - the PHANToM would provide the appropriate resistance as the needle penetrated various different layers of tissue, each with different type of resistance to penetration.

The work in progress on these two systems which is demonstrated here represents ongoing research in a range of projects. It will provide those who try out the demonstrations with the experience of feeling and seeing the possibilities of this new range of HCI technologies.

The light, hand-held locating devices are easily manipulated, with their representation as virtual tools in the virtual display being whatever is appropriate to the 


\section{DESCRIPTION}

\subsection{Virtual Workbench}

The Virtual Workbench consists of a fibreglass frame with a footprint of $900 \mathrm{~mm} \times 600 \mathrm{~mm}$ and a height of $700 \mathrm{~mm}$. A workstation monitor is mounted on top of the frame. The user sits at the frame looking at a tilted mirror which is at about shoulder height and which reflects the image on the monitor. The user's hands reach in under the mirror into the same space as the reflected monitor image appears to occupy. With shutter glasses this becomes a $3 \mathrm{D}$ image. Two radiofrequency hand held tracking devices let the user manipulate the displayed data.

The software which currently supports the Virtual Workbench runs on Silicon Graphics workstations, either an Indigo 2 or Onyx. This demonstration system uses an Indigo2 with a "Maximum Impact" graphics subsystem.

\subsection{PHANToM Haptic Device}

The PHANToM is a robotic arm sitting on a $330 \mathrm{~mm} \mathrm{x}$ $250 \mathrm{~mm}$ base. At the end of the arm is a pen-like tool which the user holds. The hardware can provide the $\mathrm{XYZ}$ location of the pen tip and the pitch, yaw and roll of the pen to the controlling software, and can respond to controlling instructions by providing in real time, a $3 \mathrm{D}$ force vector at the tip of the pen. The pen is equipped with one finger-operated button.

These features allow the PHANToM to act both as a 3D tracking/input device and as a 3D force-feedback device. With appropriate programming, the PHANToM can simulate things like a hard surface, a soft squishy surface, a rough surface and so forth. The nature of interaction with this haptic device is quite different to interactions with visual devices, and involves dynamic rather than static interaction.

\section{INDUSTRIAL APPLICATIONS}

The initial development work on the Virtual Workbench, by staff at the Institute of Systems Science at the National University of Singapore, focused on medical applications where the data sets were typically
3D reconstructions of body parts from CAT or MRI scans. The scale of the data is of the same order of magnitude as the scale of the virtual workspace in which the operator's hands sit. The nature of the data is primarily voxel, leading to very large data sets and demanding voxel resolution algorithms and texture mapping.

A second set of applications is being built around geological, mine and minerals exploration data. This data is characterised by lines (drillholes), surfaces (geological contacts, ore body boundaries, digital elevation models) and volumes (interpolated ore grade). The data items often have many different attribute sets for example drillhole data records position, lithology, assay values for many minerals as well as geophysical measurements. A large industry already exists to handle these data sets, and it is facing major obstacles in displaying and interpreting the many data sets in the true 3-dimensional context. Developments using this virtual environment technology show great promise towards satisfying the needs of professionals in this area.

\section{REFERENCES}

Massie, T and Salisbury, J.K. (1994) The PHANToM Haptic Interface: A Device for Probing Virtual Objects Proceedings of the ASME Winter Annual Meeting 295302

Poston, T and Serra, L. (1994) The Virtual Workbench: Dextrous VR. Proceedings ACM VRST'94, 111-122.

\section{ACKNOWLEDGEMENT}

The authors wish to acknowledge that this work was carried out within the Cooperative Research Centre for Advanced Computational Systems established under the Australian Government's Cooperative Research Centre Program. 